\title{
Ramp lesions: a systematic review of MRI diagnostic accuracy and treatment efficacy
}

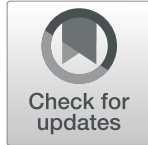

José Moreira ${ }^{1 *} \mathbb{D}$, Margarida Almeida ${ }^{1}$, Nuno Lunet $^{2,3}$ and Manuel Gutierres ${ }^{1,4}$

\begin{abstract}
Purpose: We conducted a systematic review of the published literature to assess the accuracy of Magnetic Resonance Imaging (MRI) in establishing the presence of ramp lesions (RLs) in Anterior Cruciate Ligament (ACL) deficient knees and the clinical efficacy of the surgical repair of RLs.

Methods: A comprehensive search of the MEDLINE, Web of Science and Scopus databases was performed according to PRISMA guidelines. Studies assessing MRI diagnostic accuracy for RLs or the clinical effect of RL repair in participants with $A C L$ injuries were included. Diagnostic accuracy measures were pooled and plotted in forest plots. Preoperative and at last follow-up treatment efficacy outcome measures were extracted and plotted in forest plots, for graphical comprehension.
\end{abstract}

Results: Sixteen studies met the criteria and were included. The diagnostic analysis showed a pooled sensitivity, specificity, positive and negative likelihood ratios of $65.1 \%$ ( $95 \% \mathrm{Cl}, 59.73$ to 70.42$), 91.6 \%$ (95\% Cl, 89.14 to 94.05), 2.91 $(95 \% \mathrm{Cl}, 2.38-3.55)$ and $0.53(95 \% \mathrm{Cl}, 0.44-0.64)$, respectively, with high heterogeneity $\left(\mathrm{I}^{2}\right.$ above $\left.80 \%\right)$ for all measures. Treatment analysis showed improved Lysholm Knee Score, IKDC score and laxity difference between the knees in all studies after meniscal suture repair. A separate analysis showed no differences between repair of smaller, stable, RLs with meniscal sutures and repair with abrasion and trephination only.

Conclusion: Although the results present considerable heterogeneity, MRI seems to demonstrate moderate accuracy in the diagnosis of RLs in patients with ACL tear and the surgical repair of RLS can be associated with improved overall outcomes.

Keywords: Ramp lesions, Anterior cruciate ligament, Knee instability, Magnetic resonance imaging, Meniscal repair

\section{Introduction}

Primarily described in 1983 by Hamberg et al. [30], injury to the peripheral attachment of the posterior horn of the medial meniscus (PHMM) after Anterior Cruciate Ligament (ACL) lesion (termed "Ramp Lesion", by Strobel et al. [77]) still remains an understudied topic.

The coexistence of ACL rupture and other knee injuries has been described in many studies. Acute ACL rupture is associated with a meniscal injury in over 50\% (16-82\%, in different studies) of injuries and over $80 \%$ of chronic ACL ruptures [10, 29, 35, 49, 62, 82, 83]. The

\footnotetext{
* Correspondence: jcpmoreira96@gmail.com

${ }^{1}$ Faculty of Medicine, University of Porto, Alameda Hernâni Monteiro, 4200-319 Porto, Portugal

Full list of author information is available at the end of the article
}

medial meniscus is firmly attached to the tibia and femur, allowing it to act as a knee stabilizer, preventing excessive anterior translation, especially in the ACLdeficient knee, thus being especially susceptible to injuries after ACL lesion [1, 6, 13, 17, 42, 70, 78].

Ramp Lesions (RL) are also often described as meniscocapsular separations and meniscosynovial tears [13]. Recent literature has extended the definition to include injuries of the meniscotibial ligament and peripheral longitudinal tears in the Red-Red zone of the PHMM [12, 13, $60,72,79,80]$ and different classification systems have been proposed by Thaunat et al. [79] and Seil et al. [64].

The epidemiology of RLs is still incompletely defined. Incidence ranges from $9 \%$ to $40 \%$ in many small population studies, becoming higher with chronicity of ACL

\section{Springer Open}

(ㅇ The Author(s). 2020 Open Access This article is licensed under a Creative Commons Attribution 4.0 International License, which permits use, sharing, adaptation, distribution and reproduction in any medium or format, as long as you give appropriate credit to the original author(s) and the source, provide a link to the Creative Commons licence, and indicate if changes were made. The images or other third party material in this article are included in the article's Creative Commons licence, unless indicated otherwise in a credit line to the material. If material is not included in the article's Creative Commons licence and your intended use is not permitted by statutory regulation or exceeds the permitted use, you will need to obtain permission directly from the copyright holder. To view a copy of this licence, visit http://creativecommons.org/licenses/by/4.0/. 
deficiency $[1,8,11,67,71]$. Male sex, younger age, chronic (> 6 weeks) ACL injury, increased time from injury, presence of a lateral meniscal tear and increased medial meniscal slope are significant risk factors for RLs $[44,65,73]$.

When a RL is present in an ACL-deficient knee, anterior and external rotational laxities are significantly increased, compared to isolated ACL injury. In such cases, repair of the ACL alone does not fully correct this abnormality, suggesting the importance of diagnosing and repairing the meniscal injury during ACL reconstruction [51, 70, 76]. However, clinical identification can be a troublesome situation. There are no specific clinical tests for the diagnosis of RLs and common tests for meniscal tears are not accurate in diagnosing this injury [55].

While MRI can be a reliable diagnostic modality for most meniscal pathologies [31], its sensitivity and specificity for the diagnosis of RL have been questioned by some authors, marking the need for further research, especially for a quantitative analysis of data from the existing studies $[11,38,55]$.

The general consensus is that arthroscopic evaluation is necessary to reliably assess the occurrence of a $\mathrm{RL}$ after ACL injury [13]. Standard anterolateral arthroscopy portals, even with the addition of probing, have limited accuracy, requiring insertion of the arthroscope in the posteromedial recess, using the Intercondylar (or Gillquist) view or a posteromedial portal [24, 54, 63, 72].

What to do when a RL is identified is not consensual and may depend on whether ACL injury is acute or chronic. Once they are located in a vascularized region of the meniscus, several authors have stated that shorter or more stable tears may be managed with conservative treatment following ACL reconstruction, especially in the acute setting $[57,68]$. Conversely, some authors state that acute repair is necessary since the hypermobility of the detached meniscocapsular structure delays, or even impedes, spontaneous healing $[1,4,13,75]$.

Repair options may include open repair, termed posteromedial arthrotomy [21,30], now widely replaced by other techniques, using posteromedial approaches (with a hook) or an anteromedial approach (all-inside or inside out repair techniques) [15, 60,77]. For small and stable subacute or chronic injuries, stimulation of a healing response with abrasion and trephination may be recommended [60]. The existing literature lacks a comprehensive analysis of data from the existing treatment studies, in order to clearly understand the benefit of repairing the meniscal tear.

The purpose of this systematic review was to assess the published literature with regard to the diagnosis and treatment of RLs in ACL deficient knees in order to describe the accuracy of MRI (compared to arthroscopy) in establishing the presence of a RL and the clinical efficacy of the surgical repair of RLs, by evaluating the difference between preoperative and postoperative knee scores. Our hypothesis was that MRI cannot adequately diagnose RLs and surgical repair of ramp lesion leads to improved clinical outcomes at final follow-up.

\section{Materials and methods}

The present study was conducted according to the Preferred Reporting Items for Systematic Reviews and Meta-Analyses (PRISMA) guidelines [50]. A protocol for the conduction of the review was written before the start of the study and followed until the end of the review.

\section{Study eligibility}

Types of studies: all study designs, except for case reports, ex vivo studies, reviews and technical notes, were included, without publication date, status or language restrictions.

Participants: studies were considered when they examined participants, of any age, with acute or chronic ACL rupture undergoing (or who underwent) reconstruction and at risk for or diagnosed with a concomitant RL.

Interventions and Comparisons: studies were included if they compared the diagnostic accuracy of MRI with arthroscopy (gold standard) or if they assessed the clinical effect of RL repair (through any method of repair).

Outcomes: primary outcomes considered were sensitivity, specificity and likelihood ratios (LR) for the diagnostic studies and Lysholm Knee Score, International Knee Documentation Committee (IKDC) Score and laxity difference between the affected and the non-affected knees, for the treatment analysis. Articles not presenting any of the aforementioned outcomes or without a pretreatment analysis of patients were excluded.

\section{Literature search}

Included databases were MEDLINE, Web of Science and Scopus. The last search was run on 12/01/2020 and search clauses can be found in appendix. The search terms cover a broad spectrum of meniscus and associated knee injuries, to avoid missing relevant literature. As a result of the different designations of RLs, keywords such as "ramp", "hidden", "meniscocapsular", "meniscosynovial" and "posteromedial" were included in the search clause. The use of additional limiters and filters was restricted, in order to avoid missing potentially relevant studies. The reference lists of the selected articles were also checked for relevancy.

\section{Study selection and data abstraction}

Two researchers independently screened the titles and abstracts yielded by the database searches against the inclusion criteria. Disagreements were solved by consensus. Full reports for all titles and abstracts that appeared to have met the inclusion criteria or where there was 
some uncertainty were sought. Full text reports were then screened and included if they met the inclusion criteria. Reasons for excluding papers were recorded. None of the researchers was blinded to the journal titles, authors or institutions.

Data regarding the study sample and methodology, intervention details (MRI and surgical techniques), and all reported important outcomes were systematically extracted from the included studies, following the predefined protocol.

\section{Risk of Bias assessment}

The Quality Assessment of Diagnostic Accuracy Studies (QUADAS-2) instrument was used to assess possible risk of bias in diagnostic studies, according to the Cochrane Collaboration recommendation [61]. Each of the 11 recommended quality items was judged as 'yes' or 'no', according to whether that characteristic was present. When there was insufficient detail reported in the study, that item was judged 'unclear'.

Quality of the included articles of the treatment studies was assessed using the Methodological Index for Non-Randomized Studies (MINORS) [69], a validated instrument, designed for non-randomized surgical studies, and based on 12 items, the last four specific for comparative studies. Each item was scored as "0", "1" or "2", if the item was not reported, reported but inadequate or reported and adequate, respectively.

Quality assessment was accomplished by one of the authors. Results are presented for each item, independently.

\section{Data analysis}

Sensitivity, specificity and positive and negative LRs, with corresponding 95\% confidence intervals (CI), were extracted whenever provided in the original reports, or computed with the available information. The diagnostic accuracy measures were pooled and analysed using a random effects model and plotted in forest plots. Statistical heterogeneity was quantified using the $\mathrm{I}^{2}$ statistic.

Preoperative and at last follow-up treatment efficacy outcome measures were extracted and plotted in forest plots for graphical comprehension of the results. No meta-analysis of these results was attempted, since no measures of association were provided in most studies. A separate analysis with two studies $[45,85]$ was performed to compare the efficacy of meniscal suture to abrasion and trephination only, in small $(<1.5 \mathrm{~cm})$ and stable RLs.

Unless otherwise noted, continuous variables were expressed as means and 95\% CI and categorical variables were expressed as frequencies. Standard deviations were used to estimate $95 \% \mathrm{CI}$, when $\mathrm{CI}$ were not provided.
Stata software (version 15.1) was used for the metaanalysis and to produce forest plots. A $P<0.05$ was considered statistically significant.

\section{Results \\ Literature search}

The systematic review flow chart is presented as Fig. 1. Initial search through the databases retrieved 1102 articles. A total of 16 original research articles were included in the systematic review, eight studies were included in the diagnostic analysis and nine studies were included in the treatment analysis, with one study [28] being included in both portions.

\section{Characteristics of the included studies}

The study and patient characteristics from the included studies are summarized in Table 1 . All studies were conducted in a single centre and evaluated a total of 1959 patients. Populations depicted in the studies presented a predominance of males (except in one study by Furumatsu et al. [26]) and young adults.

The MRI characteristics of the diagnostic studies included are summarized in Table 2. Hatayama et al. [41] used two cohorts in their study to compare different magnet strengths in the diagnosis of RL (3.0-Tesla versus 1.5Tesla). MRI diagnostic criteria are similar in all but one study by Kumar et al. [40], where they used oedema of the tibial plateau as a marker of RLs. Sagittal fat-suppressed proton density-weighted image and fat-suppressed T2weighted image were the preferred sequences. Only two studies [22, 32] reported MRI interpretation simultaneously by a musculoskeletal radiologist and an orthopaedic surgeon, the remaining reported MRI interpretation by either a radiologist or a surgeon only. The estimated time from injury to the diagnostic MRI was not mentioned in any of the studies.

Table 3 compiles the treatment approaches from the studies included in the review. ACL reconstruction was performed in all patients, either by a hamstring autograft (640 patients), a patellar bone-tendon-patellar bone autograft (98 patients) and a quadriceps tendon graft (two patients). The ACL reconstruction strategy was absent in three studies [28, 36, 43]. Sonnery-Cottet et al. [74] added anterolateral ligament repair to the intervention in 189 patients. All studies present different postoperative rehabilitation protocols, with many common key points. All patients were followed for a minimum of 12 months, except in the study by Gulenc et al. [28] (33 weeks).

\section{Risk of Bias assessment}

Regarding risk of bias in the diagnostic studies, portrayed in Fig. 2, all studies satisfied at least six of the 11 items recommended by the QUADAS-2 tool. Three studies were considered of low quality concerning the 


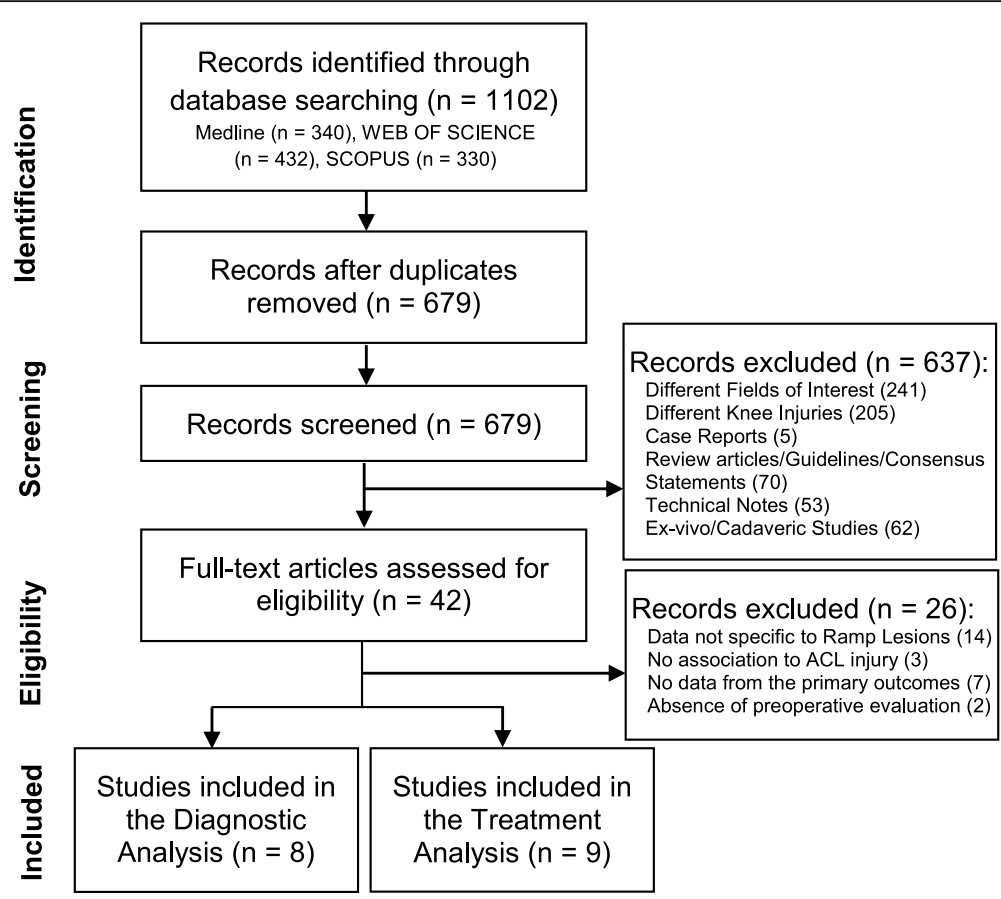

Fig. 1 Study selection process for the Systematic Review using the PRISMA (Preferred Reporting Items for Systematic Reviews and Meta-Analyses) guidelines. Gulenc et al. [28] was included in both portions of the analysis

Table 1 Study and Patient Characteristics ${ }^{\mathrm{a}}$

\begin{tabular}{|c|c|c|c|c|c|c|c|}
\hline Author (Year) & Study Period & Design & & $N$ & Age, $y^{b}$ & Male, \% & Focus \\
\hline Arner (2017) [7] & 2013 to 2015 & $\mathrm{P} / \mathrm{NC}$ & & 90 & $28 \pm 10(14-45)$ & 50.0 & $\mathrm{D}$ \\
\hline Chen (2018) [14] & Aug/2010 to Dec/2014 & $R / C$ & & 46 & $26(18-41)$ & 73.9 & $\mathrm{~T}$ \\
\hline DePhillipo (2017) [22] & April/2010 to July/2016 & $\mathrm{P} / \mathrm{C}$ & & 301 & $29.6 \pm 12.5(14-61)$ & 66.0 & $\mathrm{D}$ \\
\hline Furumatsu (2014) [26] & July/2009 to Dec/2011 & $\mathrm{P} / \mathrm{C}$ & & 20 & $19(15-38)$ & 40.0 & $\mathrm{~T}$ \\
\hline Gulenc (2019) [28] & 2017 & $\mathrm{P} / \mathrm{NC}$ & & 15 & $26.8(18-35)$ & 53.3 & $\mathrm{D} / \mathrm{T}$ \\
\hline Hatayama (2018) [32] & April/2013 to Aug/2017 & $\mathrm{P} / \mathrm{C}$ & & 155 & $25.3(13-60)$ & 51.0 & $\mathrm{D}$ \\
\hline Keyhani (2017) [36] & 2011 to 2014 & $\mathrm{P} / \mathrm{C}$ & & 128 & $24(18-48)$ & 83.6 & $\mathrm{~T}$ \\
\hline Kim (2018) [38] & June/2011 to April/2015 & $\mathrm{P} / \mathrm{C}$ & & 195 & $31.7 \pm 11.7$ & 88.2 & $\mathrm{D}$ \\
\hline Kumar (2018) [40] & Jan/2006 to June/2016 & $R / C$ & & 178 & NR. & NR. & $\mathrm{D}$ \\
\hline Li (2015) [43] & Aug/2011 to Feb/2014 & $\mathrm{P} / \mathrm{C}$ & & 23 & NR. & NR. & $\mathrm{T}$ \\
\hline \multirow[t]{2}{*}{ Liu (2017) $[45]^{c}$} & Aug/2008 to April/2012 & $\mathrm{P} / \mathrm{C}$ & (SG) & 50 & $35.6 \pm 8.5$ & 76 & $\mathrm{~T}$ \\
\hline & & & $(A G)$ & 41 & $34.8 \pm 9.1$ & 73.2 & \\
\hline Malatray (2018) [48] & Oct/2014 to May/2016 & $\mathrm{P} / \mathrm{C}$ & & 56 & $14.0 \pm 1.3(12-17)$ & 76.8 & $\mathrm{D}$ \\
\hline Sonnery-Cottet (2018) [74] & Jan/2013 and Aug/2015 & $R / C$ & & 383 & $27.4 \pm 9.2(14-60)$ & 76.5 & $\mathrm{~T}$ \\
\hline Thaunat (2016) [80] & Oct/2012 to March/2013 & $P / C$ & & 132 & $26.4(12-57)$ & 83.3 & $\mathrm{~T}$ \\
\hline \multirow[t]{2}{*}{ Yang (2017) $[85]^{c}$} & Jan/2010 to Jan/2014 & $R / C$ & $(\mathrm{SG})$ & 37 & $35.7 \pm 8.5$ & 75.7 & $\mathrm{~T}$ \\
\hline & & & (AG) & 31 & $34.8 \pm 8.1$ & 74.2 & \\
\hline Yeo (2018) [86] & Jan/2015 to Sep/2017 & $R / C$ & & 78 & $37.3(19-52)$ & 82.1 & D \\
\hline
\end{tabular}

${ }^{\mathrm{a}} A G$ abrasion and trephination group, Aug August, $D$ diagnosis, Dec December, Feb February, Jan January, NR not reported, Oct October, $P$ prospective, $R$ retrospective, Sep September, SG meniscal suture group, $T$ treatment, $Y$ years

${ }^{\mathrm{b}}$ Age is expressed as mean \pm SD (Range), when available

'Liu et al. [45] and Yang et al. [85] used 2 different cohorts to compare different treatment approaches 
Table $2 \mathrm{MRI}$ characteristics of the Studies included in this review ${ }^{\mathrm{a}}$

\begin{tabular}{|c|c|c|c|c|c|}
\hline Author (Year) & Knee Position & Magnet Strength, T & $\begin{array}{l}\text { Slice Thickness \& } \\
\text { MRI Sequence }\end{array}$ & RLs, \% & Diagnostic Criteria \\
\hline Arner (2017) [7] & Near full extension. & 1.5 & 3 mm; Sequences NR. & 14.4 & $\begin{array}{l}\text { High SI or separation between the } \\
\text { posterior capsule and the PHMM. }\end{array}$ \\
\hline DePhillipo (2017) [22] & NR. & 3.0 or 1.5 & NR; Sag. PDFS and T2FS. & 16.6 & $\begin{array}{l}\text { High SI or separation between the } \\
\text { posterior capsule and the PHMM. }\end{array}$ \\
\hline Gulenc (2019) [28] & NR. & NR. & NR; Sagittal T2FS. & NR. & $\begin{array}{l}\text { Separation between the capsule } \\
\text { and the PHMM or tibial oedema. }\end{array}$ \\
\hline \multirow[t]{2}{*}{ Hatayama (2018) [32] $]^{\mathrm{b}}$} & \multirow[t]{2}{*}{ Near full extension. } & $3.0(N=59)$ & $2 \mathrm{~mm}$; Sag. PDFS. & 20.3 & \multirow{2}{*}{$\begin{array}{l}\text { High SI or separation between the } \\
\text { posterior capsule and the PHMM. }\end{array}$} \\
\hline & & $1.5(N=96)$ & NR. & 37.8 & \\
\hline Kim (2018) [38] & NR. & NR. & NR; Sag. PDFS. & 25.6 & $\begin{array}{l}\text { Peripheral } \mathrm{LT} \leq 4 \mathrm{~mm} \text { of the } \\
\text { meniscocapsular junction of the PHMM }\end{array}$ \\
\hline Kumar (2018) [40] & NR. & NR. & NR; Sag. PDFS and T2FS. & 14.9 & $\begin{array}{l}\text { Oedema of the posterior medial } \\
\text { tibial plateau. }\end{array}$ \\
\hline Malatray (2018) [48] & Near full extension. & NR. & NR. & 23.2 & $\begin{array}{l}\text { Peripheral LT of the meniscocapsular } \\
\text { junction of the PHMM. }\end{array}$ \\
\hline Yeo (2018) [86] & Neutral & 3.0 or 1.5 & 3-4 mm; Sag. PDFS and T2FS. & 9.0 & $\begin{array}{l}\text { High SI or separation between the } \\
\text { posterior capsule and the PHMM. }\end{array}$ \\
\hline
\end{tabular}

${ }^{a} L T$ longitudinal tear, MRI Magnetic Resonance Imaging, NR not reported, PDFS Fat-suppressed Proton Density-weighted image, PHMM posterior horn of the Medial Meniscus, RLs proportion of ramp lesions, Sag Sagittal, SI fluid-like Signal Intensity, TFI time from injury, T2FS fat-suppressed T2-weighted image, T Tesla ${ }^{b}$ Hatayama et al. [32] used 2 cohorts to compare different magnet strengths in the diagnosis of ramp lesions

representativeness of the spectrum of patients, as a result of the study of a paediatric population [48] or the study of patients already diagnosed with Ramp Lesions $[22,28]$. The interval between MRI and the reference standard was absent in three studies [38, 40, 48].
Blinding of the two tests results was only reported in three studies [7, 22, 40] and only one study [86] reported on the clinical information available at the time of interpretation of test results. All other topics were considered of high quality for every study.

Table 3 Treatment Methods from the Studies included in this review ${ }^{a}$

\begin{tabular}{|c|c|c|c|c|c|}
\hline Author (Year) & Surgery Details \& ACL Graft & Postoperative Protocol & $\begin{array}{l}\text { TFI to } \\
\text { Repair }\end{array}$ & $\begin{array}{l}\text { Follow-up } \\
\text { Time }\end{array}$ & $\begin{array}{l}\text { Adverse } \\
\text { Events }\end{array}$ \\
\hline Chen (2018) [14] & $\begin{array}{l}\text { All-inside suture device } \\
\text { (FasT-Fix). HT. }\end{array}$ & $\begin{array}{l}0^{\circ}-90^{\circ} \text { at } 4 \text { wks; full WB/ROM in } 6 \text { wk.; } \\
\text { full activity at } 6 \text { mo. }\end{array}$ & NR. & $32 \mathrm{mo}$. & $\begin{array}{l}2 \text { MFC cartilage } \\
\text { injuries. }\end{array}$ \\
\hline Furumatsu (2014) [26] & $\begin{array}{l}\text { All-inside suture device } \\
\text { (FasT-Fix). BPTB, HT. }\end{array}$ & $\begin{array}{l}\text { Partial WB in } 2 \text { wk.; full WB in } 4-6 \text { wk.; } \\
\text { full activity in } 5-8 \text { mo. }\end{array}$ & $6 \mathrm{mo}$ & $24 \mathrm{mo}$. & $\begin{array}{l}5 \% \text { secondary } \\
\text { interventions. }\end{array}$ \\
\hline Gulenc (2019) [28] & $\begin{array}{l}\text { All-inside suture technique. } \\
\text { NR. }\end{array}$ & $\begin{array}{l}0-90^{\circ} \text { by the } 3 r d \text { wk.; } \\
\text { full activity in } 4-6 \text { mo. }\end{array}$ & NR. & $33.1 \pm 12.7 w k$ & NR. \\
\hline Keyhani (2017) [36] & $\begin{array}{l}\text { All-inside suture with hook. } \\
\text { NR. }\end{array}$ & $\begin{array}{l}0^{\circ}-90^{\circ} \text { and partial WB after } 2-4 w k \text {.; } \\
\text { full WB and ROM at } 6 \text { wk. }\end{array}$ & NR. & $>24 \mathrm{mo}$ & $\begin{array}{l}\text { Residual joint } \\
\text { pain in } 3 \text { pts. }\end{array}$ \\
\hline Li (2015) [43] & $\begin{array}{l}\text { All-inside suture device } \\
\text { (FasT-Fix). NR. }\end{array}$ & $\begin{array}{l}0^{\circ}-90^{\circ} \text { by the } 4 \text { th } w k \text {; full WB in } 6 \text { wk.; } \\
\text { full activity after } 6 \text { mo. }\end{array}$ & NR. & 14 mo. & NR. \\
\hline Liu (2017) [45] & $\begin{array}{l}\text { All-inside suture with hook. } \\
\text { HT. }\end{array}$ & $\begin{array}{l}0^{\circ}-90^{\circ} \text { by the } 4 \text { th wk.; full WB at } 4 \text { wk.; } \\
\text { full activity at } 9-12 \text { mo. }\end{array}$ & NR. & $37.9 \pm 15.9 \mathrm{mo}$ & NR. \\
\hline Sonnery-Cottet (2018) [74] & $\begin{array}{l}\text { All-inside suture with hook. } \\
\text { BPTB, HT. }\end{array}$ & $\begin{array}{l}0^{\circ}-90^{\circ} \text { by the } 4 \text { th } w \text { k.; WB as tolerated; } \\
\text { full activity at } 8-9 \text { mo. }\end{array}$ & $13.5 \pm 32 \mathrm{mo}$ & $37.4 \pm 9 \mathrm{mo}$ & NR. \\
\hline Thaunat (2016) [80] & $\begin{array}{l}\text { All-inside suture with hook. } \\
\text { HT, BPTB, QT. }\end{array}$ & $\begin{array}{l}0^{\circ}-90^{\circ} \text { by the } 6 \text { th wk.; full WB in } 3 \text { wk.; } \\
\text { full activity at } 9 \text { mo. }\end{array}$ & $N R$ & $27 \mathrm{mo}$. & $\begin{array}{l}2 \text { hematomas } \\
\text { needing lavage. }\end{array}$ \\
\hline Yang (2017) [85] & $\begin{array}{l}\text { All-inside suture device } \\
\text { (FasT-Fix). HT. }\end{array}$ & $\begin{array}{l}\text { Partial WB at } 8 \text { wks; full WB at } 12 \text { wk.; } \\
\text { full activity after } 6 \text { mo. }\end{array}$ & $45.2 \pm 28.1 \mathrm{~d}$ & $>24 \mathrm{mo}$ & $\begin{array}{l}\text { Residual joint } \\
\text { pain in } 3 \text { pts. }\end{array}$ \\
\hline Liu (2017) [45] & Abrasion and trephination. $\mathrm{HT}$. & $\begin{array}{l}0^{\circ}-90^{\circ} \text { by the } 4 \text { th wk.; full WB at } 4 \text { wk.; } \\
\text { full activity at } 9-12 \text { mo. }\end{array}$ & NR. & $40.3 \pm 16.5 \mathrm{mo}$ & NR. \\
\hline Yang (2017) [85] & Abrasion and trephination. $\mathrm{HT}$. & $\begin{array}{l}\text { Partial WB at } 8 \text { wks; full WB at } 12 \text { wk.; } \\
\text { full activity after } 6 \text { mo. }\end{array}$ & $42.8 \pm 25.4 \mathrm{~d}$ & $>24 \mathrm{mo}$ & $\begin{array}{l}\text { Residual joint } \\
\text { pain in } 2 \text { pts. }\end{array}$ \\
\hline
\end{tabular}

${ }^{a} A C L$ Anterior Cruciate Ligament, BTB bone-tendon-bone autograft, $d$ days, MFC medial femoral condyle, mo months, $H T$ hamstring tendon autograft, NR not reported, pts patients, QT quadriceps tendon autograft, ROM range of motion, TFI time from injury, WB weight-bearing, wk weeks 


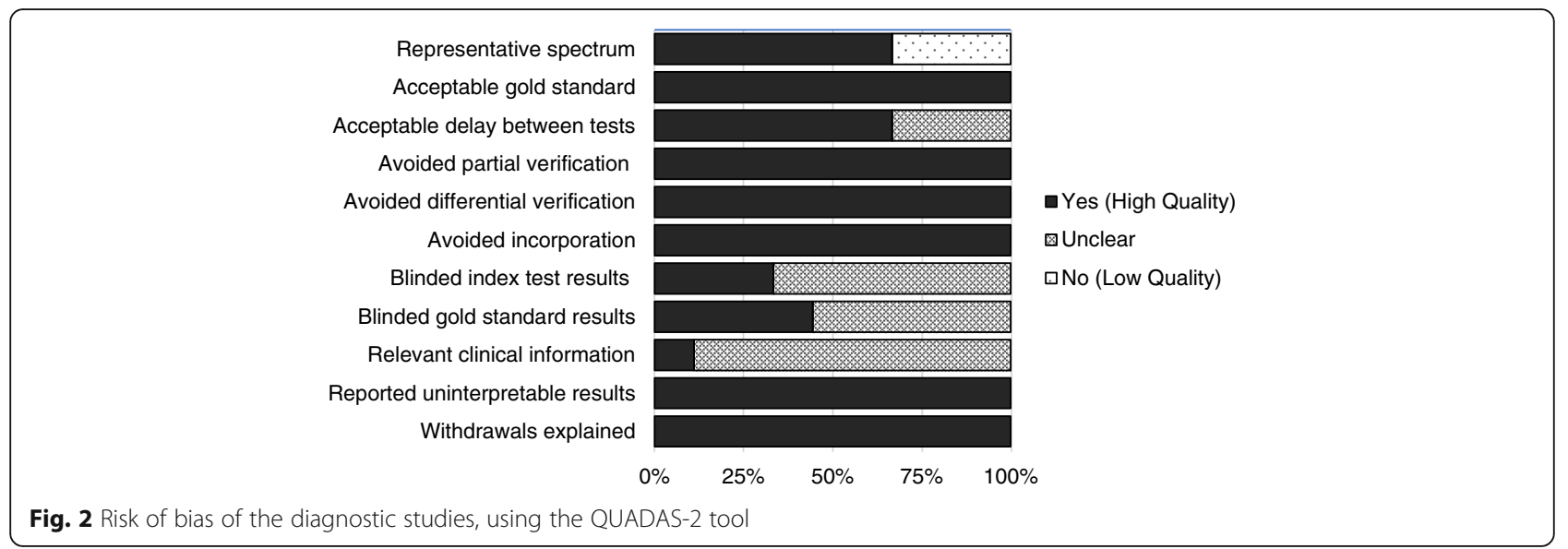

Table 4 summarizes risk of bias in the treatment studies according to the MINORS tool. Liu et al. [45] was not included in the quality assessment, as it was designed as a randomized controlled trial. This study was assessed to have a low overall risk of bias, according to the randomization process, blinding of the allocated intervention and unbiased outcome measurements. Blinding of the interventions to the investigators assessing the outcomes was only performed in one study, by Sonnery-Cottet et al. [74], as was the case for prospective calculation of the study sample, performed only in the study by Keyhani et al. [36].

\section{Diagnostic accuracy of MRI}

Figure 3 depicts the forest plots summarizing the accuracy of MRI in the detection of RLs. The pooled results showed a sensitivity of $65.08 \%$ ( $95 \%$ CI, 59.73 to 70.42 ), a specificity of $91.59 \%$ (95\% CI, 89.14 to 94.05$)$, a positive LR of 2.91 (95\% CI, 2.38 to 3.55) and a negative LR of 0.53 (95\% CI, 0.44 to 0.64 ). Heterogeneity was high, with $\mathrm{I}^{2}$ statistics above $80 \%$ for all outcomes evaluated.

\section{Treatment efficacy of ramp lesion repair}

Figure 4 shows the forest plots describing the results from studies that evaluated the effects of treatment. Mean preoperative and final Lysholm Knee Scores ranged from 56.8 to 68.6 and 84.5 to 94.4 , respectively. Mean preoperative and final IKDC scores ranged from 52.7 to 64.3 and 82.1 to 90.6 , respectively. Mean preoperative and final laxity differences between the affected and the unaffected knees ranged from $6.1 \mathrm{~mm}$ to $7.2 \mathrm{~mm}$ and $0.4 \mathrm{~mm}$ to $1.6 \mathrm{~mm}$, respectively. The improved final outcomes are statistically significant in all studies $(P<0.05)$, using tests for paired samples.

Figure 5 presents the comparison of the all-inside suture technique of the medial meniscus versus abrasion and trephination for the repair of small and stable Ramp
Lesions $(<1.5 \mathrm{~cm})$, in the two studies that evaluated both techniques. Lysholm Knee Scores, IKDC scores and laxity differences between the affected and the unaffected knees in both groups increased significantly postoperatively $(P<0.05)$, but no significant differences were observed between the two groups before or after the surgery $(P>0.05)$ in both studies.

\section{Discussion}

The results of this review demonstrated that MRI has a moderate sensitivity (65\%) and a high specificity (92\%) in the diagnosis of RL. The positive and negative LR (2.91 and 0.53 , respectively) indicate a questionable clinical significance of the MRI, as the pre-test probability will only suffer slight (around 15\%) modifications after MRI interpretation.

MRI has been appointed as a reliable diagnostic modality for most medial meniscal pathologies, with sensitivities of over $90 \%$ and specificities of over $80 \%$, in two systematic reviews with meta-analysis [18, 31, 53]. This accuracy for the diagnosis of medial meniscus injury is said to be lower in the presence of an ACL tear [20,52], which may explain the lower sensitivity of MRI for the diagnosis of RLs found in this review. DePhillipo et al. [23] inquired 36 directors of orthopaedic sports medicine through an electronic questionnaire and found that despite $89 \%$ of surgeons stated that they routinely use MRI for the diagnosis, $50 \%$ believed that they are rarely or only sometimes accurate in the diagnosis [23]. In fact, our results suggest that MRI may have a good accuracy in the diagnosis of RLs, but arthroscopy remains the reference standard and should not be replaced by MRI, as stated in the literature for other cartilage damages in the knee $[18,25,53]$.

Our results showed that Lysholm Knee Scores, IKDC scores and laxity difference between the affected and the unaffected knees significantly improve after RL repair 


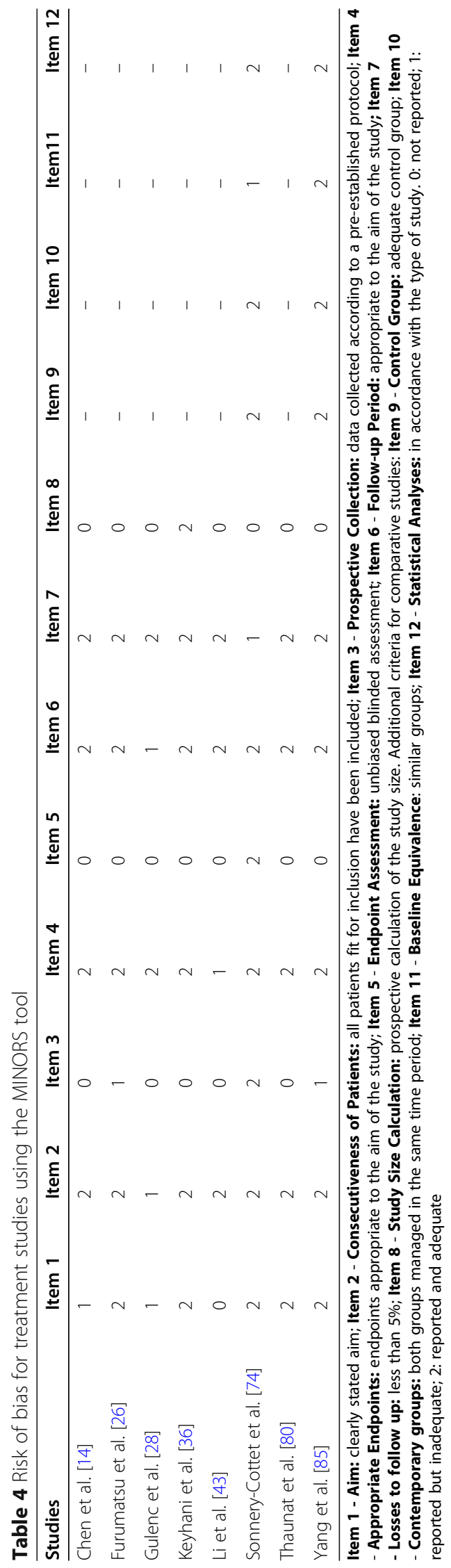




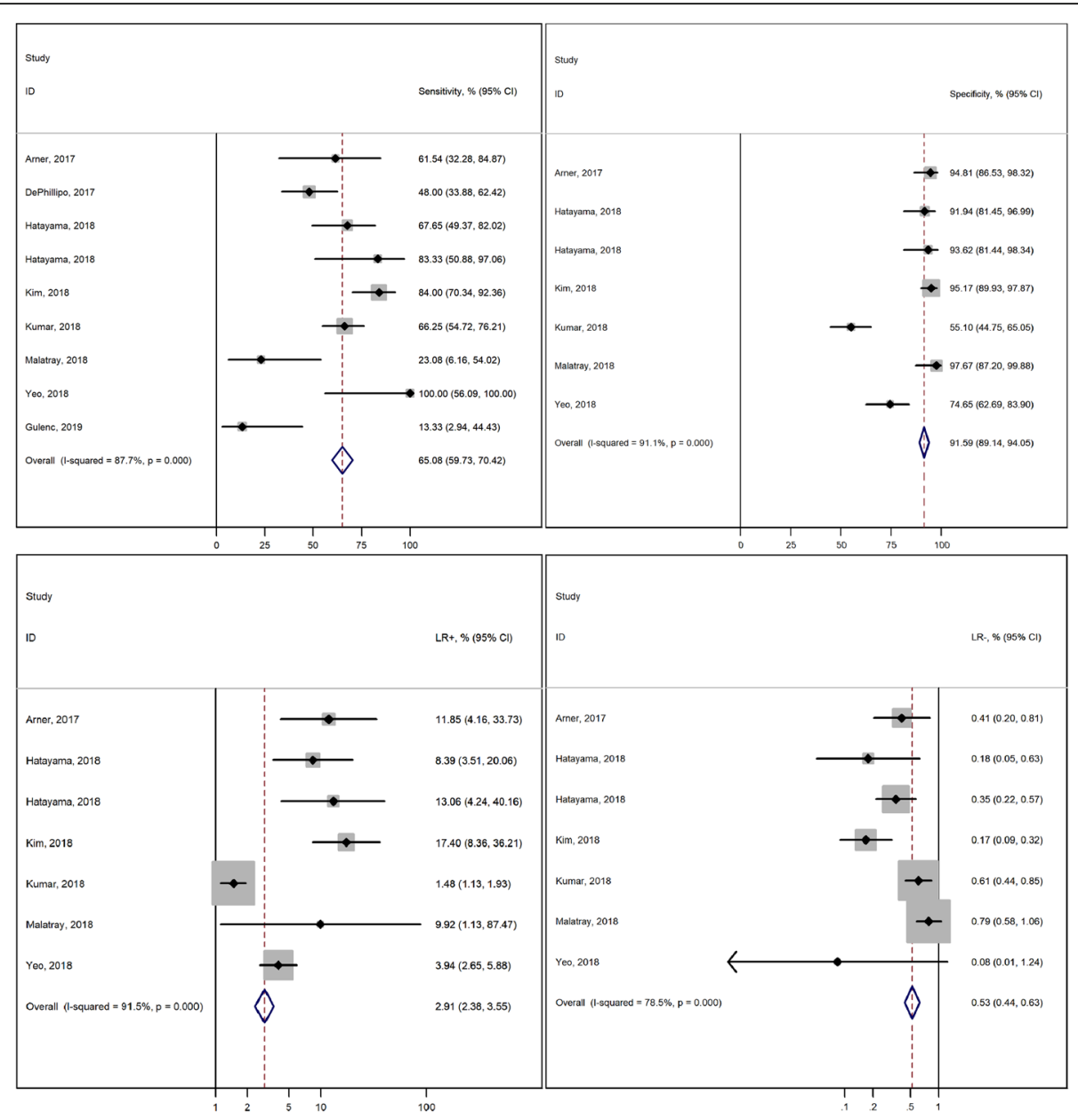

Fig. 3 Forest plots summarizing MRI accuracy in the detection of ramp lesions. Dots in squares represent the estimated measures while the horizontal lines represent the $95 \% \mathrm{Cl}$. The diamond shape represents the combined estimate. $I^{2}$ with $95 \% \mathrm{Cl}$ and the result of the using the chi-squared test are also provided. Hatayama et al. [32] used 2 different cohorts to compare different magnet strengths, 3-Tesla (upper) and 1,5-Tesla (lower)

with sutures. The two studies which compared all-inside suture and abrasion and trephination of the meniscus in small and stable RLs $(<1.5 \mathrm{~cm})$ found no significant differences between the two methods, suggesting that in these cases, abrasion and trephination may be a viable option for the management of RLs [45, 85].

Medial meniscus repair associated with ACL repair has been associated with lower rates of meniscectomy and osteoarthritis $[16,46,66]$. Moreover, repair of injuries to the PHMM in the context of ACL reconstruction has been associated with high success rates, when evaluated by second-look arthroscopy (complete healing ranging from 82.1 to $96.4 \%$ ), with little complications and satisfactory clinical results [3, 4]. Results from this review showed that the surgical repair leads to improved clinical results compared to preoperative scores, congruent with the results from medial meniscal repair of other injuries. Despite the absence of a meta-analysis of these results, because no effect measures for direct comparisons between the pre and post treatment periods were provided in the original reports, the visual presentation of the results in forest plots provides a good picture of the benefit of surgery and differs from previous reviews $[5,12]$.

It is generally accepted that extensive medial meniscal injuries require surgical repair (with inside-out or allinside sutures) and $92 \%$ of surgeons reported to surgically repair meniscal RLs in their clinical practice [23]. On the other hand, there is some controversy in the management of small $(<1.5-2 \mathrm{~cm})$ and stable (with little anterior translation of the PHMM from the anteromedial portal) meniscal tears $[58,68,84]$. In the two studies included in this review, comparing all-inside sutures to abrasion and trephination of the meniscus, the overall outcomes were similar, but larger population studies are warranted. 

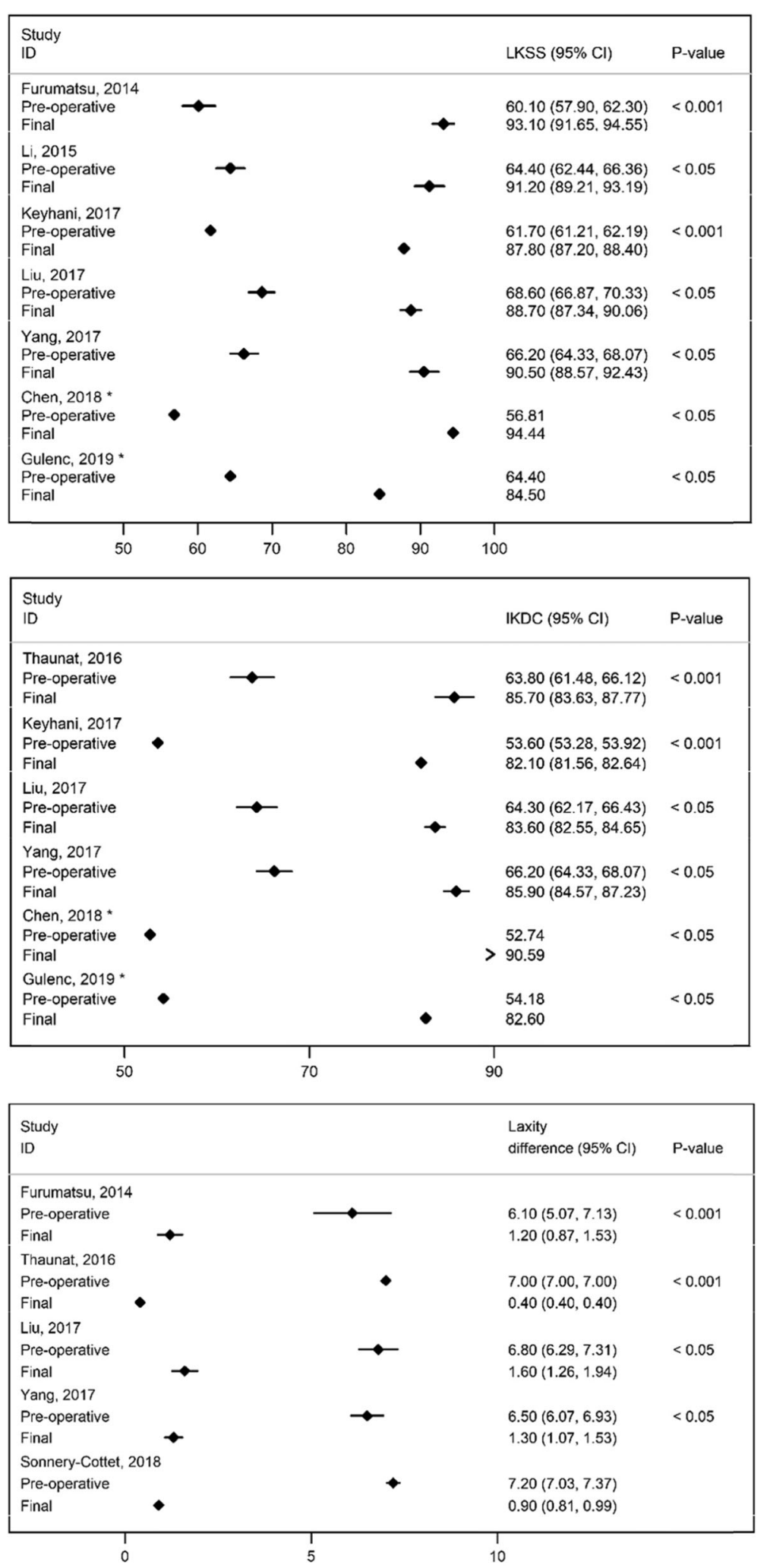

Fig. 4 Forest plots grouping the mean Pre-operative and Final (at final follow-up) Lysholm Knee Scores, International Knee Documentation Committee scores and laxity differences between the affected and the unaffected knee. Dots in squares represent the estimated measures while the horizontal lines represent the 95\% Cl. *only point estimates are presented because no confidence intervals or information to compute them were available from these studies 

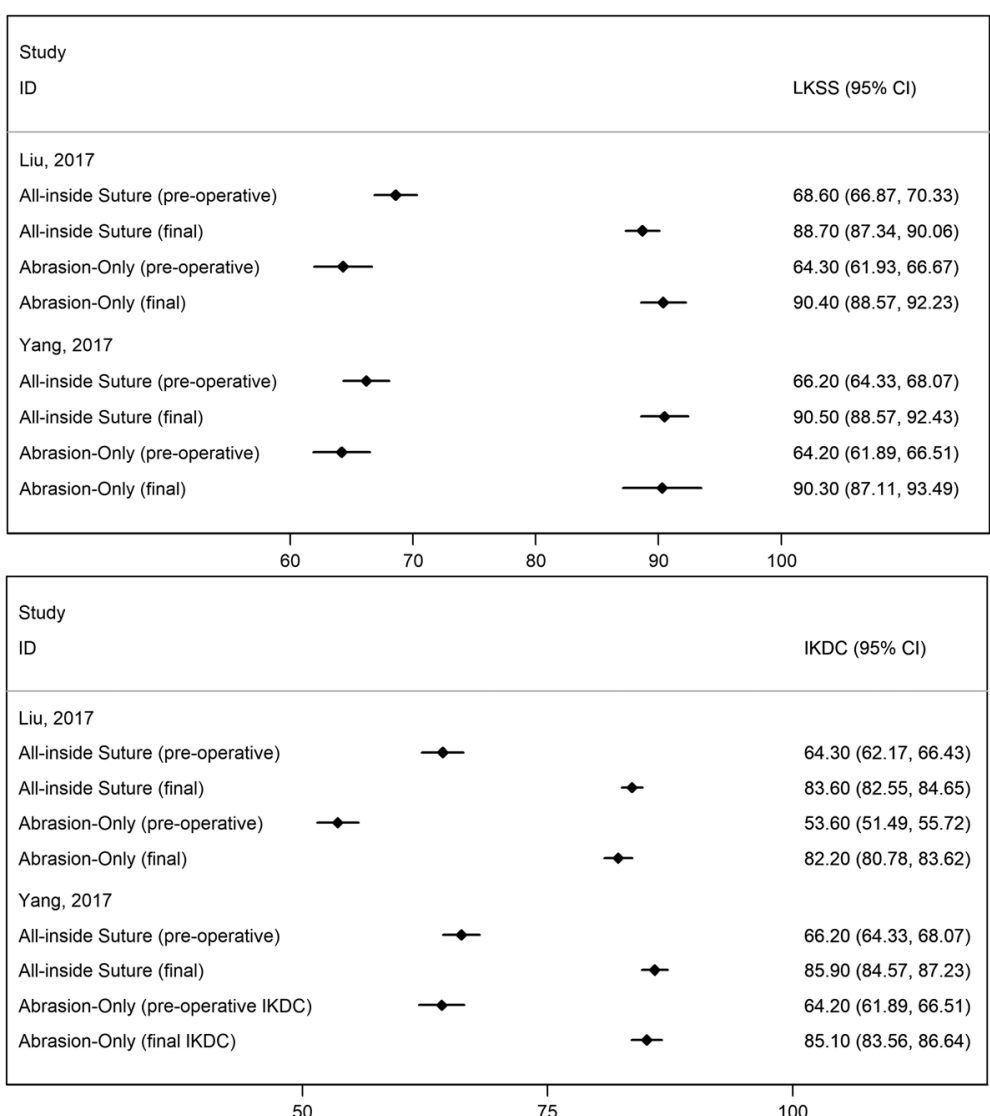

\begin{tabular}{|c|c|c|c|}
\hline \multicolumn{3}{|l|}{ Study } & \multirow{2}{*}{$\begin{array}{l}\text { Laxity } \\
\text { difference }(95 \% \mathrm{Cl})\end{array}$} \\
\hline ID & & & \\
\hline \multicolumn{4}{|l|}{ Liu, 2017} \\
\hline All-inside Suture (pre-operative) & & $\rightarrow$ & $6.80(6.29,7.31)$ \\
\hline All-inside Suture (final) & 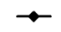 & & $1.60(1.26,1.94)$ \\
\hline Abrasion-Only (pre-operative) & & $\longrightarrow$ & $7.10(6.47,7.73)$ \\
\hline Abrasion-Only (final) & $\rightarrow$ & & $1.50(1.15,1.85)$ \\
\hline \multicolumn{4}{|l|}{ Yang, 2017} \\
\hline All-inside Suture (pre-operative) & & $\rightarrow$ & $6.50(6.07,6.93)$ \\
\hline All-inside Suture (final) & 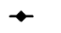 & & $1.30(1.07,1.53)$ \\
\hline Abrasion-Only (pre-operative laxity diff & & $\multimap$ & $6.70(6.19,7.21)$ \\
\hline Abrasion-Only (final laxity difference) & $\rightarrow$ & & $1.40(1.07,1.73)$ \\
\hline
\end{tabular}

Fig. 5 Forest plots comparing the mean Preoperative and Final (at final follow-up) outcomes between all-inside suture of the medial meniscus versus abrasion and trephination for the repair of small and stable Ramp Lesions $(<1.5 \mathrm{~cm})$, in the two studies that evaluated both techniques. Dots in squares represent the estimated measures while the horizontal lines represent the $95 \% \mathrm{Cl}$

\section{Limitations}

The present systematic review has a few limitations that should be discussed. This review analyses data of a relatively small number of studies. Regardless of the comprehensiveness of the search expressions, the use of multiple databases and the inclusion of articles in several languages, the available literature on this topic is scarce and some of the articles failed to report important outcomes (such as, sensitivity and specificity for diagnostic studies [8, 11, 37, 39, 72] and preoperative plus postoperative clinical outcomes [2, 34, 41, 73] for treatment studies) and had to be excluded.

Both the diagnostic and treatment studies included are heterogeneous regarding the methods used. Different magnet strengths, different knee position, differences in the diagnostic criteria and differences in the arthroscopic protocol could be responsible for the differences in sensitivity and specificity and for the heterogeneity 
encountered, in the diagnostic studies. The evidence regarding the accuracy of different magnet strengths in the diagnosis of meniscal injuries is conflicting [27, 47, 59, 81], and although 3-Tesla MRI appears to be superior to 1.5-Tesla, a recent meta-analysis showed no statistically significant difference between the two resolutions in sensitivity and specificity [56]. There are no defined criteria to diagnose RLs on MRI, but irregular posterior meniscal outline and fluid separating the meniscus and capsule, are considered to correlate best with the diagnosis of RLs [19,31] and may explain the conflicting results found by Kumar et al. [40]. Considering patient position, Bollen [11] hypothesized that when the knee is in near full extension, meniscocapsular separation is reduced, making the diagnosis harder and affecting the sensitivity of MRIs. Finally, all studies reported arthroscopy as the gold-standard for the diagnosis of RLs, but the arthroscopic protocol was heterogenous. Two studies reported $[22,40]$ assessment of RLs by probing from anterolateral portals, while two studies $[28,32]$ reported adding a posteromedial portal to the protocol if an injury was suspected during probing from the anterolateral portal and three studies [7, 38, 48] reported using posteromedial portals in every patient (Yeo et al. [86] did not specify the portals used during arthroscopy). Although, the evidence regarding the accuracy of different portals in the diagnosis of RLs during arthroscopy is conflicting [24, $38,44,72]$, the adoption of different arthroscopic protocols by the studies may have affected our findings.

In the treatment studies, differences between the surgery and postoperative protocols could also be responsible for some variability in the results. To our knowledge, no study has compared the efficacy of allinside suture using a device (anteromedial approach) with all-inside suture using a hook (posteromedial approach). Visual inspection of the forest plot conveys the impression that outcomes between the two methods are similar, but a more objective approach, with direct comparison of the two methods, is important and missing in the literature. Sonnery-Cottet et al. [74] performed anterolateral ligament reconstruction in 189 patients, but no significant differences were found between the two groups, regarding clinical scores. Also, there was variability between the postoperative rehabilitation protocols adopted by each article and, even though most share the same basic principles, as prevention of excessive weightbearing and joint compressive forces (that lead to disruption of meniscal healing $[9,33,55,75]$ ), a standardization of the postoperative protocol is needed for future research.

The studies included in this review were also heterogeneous regarding the amount of information provided. Mean time from injury to the diagnostic MRI was absent in all the diagnostic studies and time from MRI to arthroscopy was missing in three [38, 40, 48]. RLs may heal spontaneously, causing a mismatch between the MRI and arthroscopic findings if there is substantial delay between the two methods. The amount of clinical information available to the radiologist at the time of MRI interpretation was omitted in most articles. As combination of clinical and MRI findings provides the most accurate non-invasive method currently available for diagnosing injuries of the menisci [18], this information is crucial and should be reported in future studies. Time from injury to surgery (and distinction between acute or chronic injuries) was also absent in many treatment studies. As chronicity of the lesion can be a factor in the decision of treatment, this information must also be provided in future studies.

The studies that addressed the effects of treatment present many quality issues. Most studies [14, 28, 36, 43, 80] were uncontrolled before-after studies with a single preoperative outcome measurement, presenting a serious risk of bias, as the observed improvements cannot be reliably attributed to the intervention, instead of other factors, as the natural history of meniscal healing. The absence of blinding was also common across the reviewed studies and contributes to an increased risk of bias.

Thus, further radiological studies are warranted using standardized optimal conditions (as knee positioning and MRI sequences evaluated) and the inclusion of clinical findings in the evaluation of the images, possibly leading to the development of preoperative diagnostic algorithms. Also, further studies comparing different surgical options and the non-surgical management of these injuries are warranted to make assertions regarding the correct approach in the management of these conditions.

\section{Conclusion}

Notwithstanding the longevity of recognition of RLs, risk factors for developing this type of injury, the incidence, diagnosis and the outcomes of treatment remain incompletely defined. Although the results present considerable heterogeneity and the quality could be improved, MRI seems to demonstrate moderate accuracy in the diagnosis of RLs in patients presenting with acute or chronic ACL tear and the surgical repair of can be associated with improved overall outcomes. A continued interest in the development of knowledge of this condition is essential.

\section{Appendix}

\section{Database query string for PubMed}

(tibial meniscus injuries[Mesh] AND ("ramp" OR "hidden" OR "meniscocapsular" OR "meniscosynovial" OR "posteromedial" OR ("medial" AND "peripheral"))) OR (("Anterior Cruciate Ligament Injuries"[Mesh] OR "meniscus"[Tiab] OR “meniscal"[Tiab]) AND ("ramp"[Tiab] OR "hidden"[Tiab]) 
AND “lesion"[Tiab]) OR ("meniscocapsular"[Tiab] OR "meniscosynovial" [Tiab] OR (("meniscus"[Tiab] OR "meniscal"[Tiab]) AND (("peripheral"[Tiab] AND "medial”[Tiab]) OR "posteromedial"[Tiab])) AND (lesion[Tiab] OR "tear"[Tiab] OR "separation"[Tiab])).

\section{Database query string for Scopus and Web of Science} TITLE-ABS-KEY ((ramp AND lesion) OR (hidden AND lesion) AND (meniscus OR meniscal OR (Anterior AND Cruciate AND Ligament))) OR (meniscocapsular OR meniscosynovial OR ((meniscus OR meniscal) AND posteromedial) AND (separation OR tear OR lesion OR injury)).

\section{Abbreviations}

ACL: Anterior Cruciate Ligament; Cl: Confidence Interval; IKDC: International Knee Documentation System; LR: Likelihood Ratio; MINORS: Methodological Index for Non-randomized Studies; MRI: Magnetic Resonance Imaging: PHMM: Posterior horn of the Medial Meniscus; PRISMA: Preferred Reporting Items for Systematic Reviews and Meta-Analyses; QUADAS-2: Quality Assessment of Diagnostic Accuracy Studies; RL: Ramp lesion

\section{Authors' contributions}

All authors contributed to the study conception and design. Literature search was performed by José Moreira and Margarida Almeida and data analysis was performed by José Moreira and Nuno Lunet. The first draft of the manuscript was written by José Moreira and all authors commented on previous versions of the manuscript. All authors read and approved the final manuscript.

\section{Funding}

The authors report no relevant sources of funding for the conducting of this review.

\section{Competing interest}

The authors declare that they have no competing interests.

\section{Author details}

${ }^{1}$ Faculty of Medicine, University of Porto, Alameda Hernâni Monteiro, 4200-319 Porto, Portugal. ${ }^{2}$ EPIUnit-Instituto de Saúde Pública, University of Porto, Porto, Portugal. ${ }^{3}$ Departamento de Ciências da Saúde Pública e Forenses e Educação Médica, Faculty of Medicine, University of Porto, Porto, Portugal. ${ }^{4}$ Serviço de Ortopedia e Traumatologia, Centro Hospitalar de S. João, Porto, Portugal.

Received: 4 August 2020 Accepted: 9 September 2020 Published online: 25 September 2020

\section{References}

1. Ahn JH, Bae TS, Kang K-S, Kang SY, Lee SH (2011) Longitudinal tear of the medial meniscus posterior horn in the anterior cruciate ligament-deficient knee significantly influences anterior stability. Am J Sports Med 39:21872193

2. Ahn JH, Kim SH, Yoo JC, Wang JH (2004) All-inside suture technique using two posteromedial portals in a medial meniscus posterior horn tear. Arthroscopy 20:101-108

3. Ahn JH, Lee YS, Yoo JC, Chang MJ, Koh KH, Kim MH (2010) Clinical and second-look arthroscopic evaluation of repaired medial meniscus in anterior cruciate ligament-reconstructed knees. Am J Sports Med 38:472-477

4. Ahn JH, Wang JH, Yoo JC (2004) Arthroscopic all-inside suture repair of medial meniscus lesion in anterior cruciate ligament-deficient knees: results of second-look arthroscopies in 39 cases. Arthroscopy 20:936-945

5. Alessio-Mazzola M, Lovisolo S, Capello AG, Zanirato A, Chiarlone F, Formica $M$, et al. Management of ramp lesions of the knee: a systematic review of the literature. Musculoskelet Surg. 2019;https://doi.org/10.1007/s12306-01900624-z. https://doi.org/10.1007/s12306-019-00624-z. Accessed 2019/10/08
6. Allen CR, Wong EK, Livesay GA, Sakane M, Fu FH, Woo SL-Y (2000) Importance of the medial meniscus in the anterior cruciate ligamentdeficient knee. J Orthop Res 18:109-115

7. Arner JW, Herbst E, Burnham JM, Soni A, Naendrup J-H, Popchak A et al (2017) MRI can accurately detect meniscal ramp lesions of the knee. Knee Surg Sports Traumatol Arthrosc 25:3955-3960

8. Balazs GC, Greditzer HG, Wang D, Marom N, Potter HG, Marx RG et al (2019) Ramp lesions of the medial meniscus in patients undergoing primary and revision $\mathrm{ACL}$ reconstruction: prevalence and risk factors. Orthop J Sports Med 7

9. Barber FA, Click SD (1997) Meniscus repair rehabilitation with concurrent anterior cruciate reconstruction. Arthroscopy 13:433-437

10. Bellabarba C, Bush-Joseph CA, Bach BR Jr (1997) Patterns of meniscal injury in the anterior cruciate-deficient knee: a review of the literature. Am J Orthop (Belle Mead NJ) 26:18-23

11. Bollen SR (2010) Posteromedial meniscocapsular injury associated with rupture of the anterior cruciate ligament: a previously unrecognised association. J Bone Joint Surg Br 92-B:222-223

12. Bumberger A, Koller U, Hofbauer M, Tiefenboeck TM, Hajdu S, Windhager R et al (2020) Ramp lesions are frequently missed in ACL-deficient knees and should be repaired in case of instability. Knee Surg Sports Traumatol Arthrosc 28:840-854

13. Chahla J, Dean CS, Moatshe G, Mitchell JJ, Cram TR, Yacuzzi C et al (2016) Meniscal ramp lesions: anatomy, incidence, diagnosis, and treatment. Orthop J Sports Med 4

14. Chen Z, Li WP, Yang R, Song B, Jiang C, Hou JY et al (2018) Meniscal ramp lesion repair using the FasT-fix technique: evaluating healing and patient outcomes with second-look arthroscopy. J Knee Surg 31:710-715

15. Choi NH, Kim TH, Victoroff BN (2009) Comparison of arthroscopic medial meniscal suture repair techniques: inside-out versus all-inside repair. Am J Sports Med 37:2144-2150

16. Claes S, Hermie L, Verdonk R, Bellemans J, Verdonk P (2013) Is osteoarthritis an inevitable consequence of anterior cruciate ligament reconstruction? A meta-analysis. Knee Surg Sports Traumatol Arthrosc 21:1967-1976

17. Clark CR, Ogden JA (1983) Development of the menisci of the human knee joint. Morphological changes and their potential role in childhood meniscal injury. J Bone Joint Surg Am 65:538-547

18. Crawford R, Walley G, Bridgman S, Maffulli N (2007) Magnetic resonance imaging versus arthroscopy in the diagnosis of knee pathology, concentrating on meniscal lesions and $\mathrm{ACL}$ tears: a systematic review. $\mathrm{Br}$ Med Bull 84:5-23

19. De Maeseneer M, Shahabpour M, Vanderdood K, Van Roy F, Osteaux M (2002) Medial meniscocapsular separation: MR imaging criteria and diagnostic pitfalls. Eur J Radiol 41:242-252

20. De Smet AA, Graf BK (1994) Meniscal tears missed on MR imaging: relationship to meniscal tear patterns and anterior cruciate ligament tears. AJR Am J Roentgenol 162:905-911

21. DeHaven KE, Black KO, Griffiths HJ (1989) Open meniscus repair: technique and two to nine year results. Am J Sports Med 17:788-795

22. DePhillipo NN, Cinque ME, Chahla J, Geeslin AG, Engebretsen L, LaPrade RF (2017) Incidence and detection of meniscal ramp lesions on magnetic resonance imaging in patients with anterior cruciate ligament reconstruction. Am J Sports Med 45:2233-2237

23. DePhillipo NN, Engebretsen L, LaPrade RF (2019) Current trends among US surgeons in the identification, treatment, and time of repair for medial meniscal ramp lesions at the time of ACL surgery. Orthop J Sports Med 7

24. Di Vico G, Di Donato SL, Balato G, Correra G, D'Addona A, Maffulli N et al (2017) Correlation between time from injury to surgery and the prevalence of ramp and hidden lesions during anterior cruciate ligament reconstruction. A new diagnostic algorithm. Muscles Ligaments Tendons J 7:491-497

25. Friemert BY, Oberländer Y, Schwarz W, Häberle H, Bähren W, Gerngross H et al (2004) Diagnosis of chondral lesions of the knee joint: can MRI replace arthroscopy? Knee Surg Sports Traumatol Arthrosc 12:58-64

26. Furumatsu T, Miyazawa S, Tanaka T, Okada Y, Fujii M, Ozaki T (2014) Postoperative change in medial meniscal length in concurrent all-inside meniscus repair with anterior cruciate ligament reconstruction. Int Orthop 38:1393-1399

27. Grossman JW, De Smet AA, Shinki K (2009) Comparison of the accuracy rates of 3-T and 1.5-T MRI of the knee in the diagnosis of meniscal tear. AJR Am J Roentgenol 193:509-514 
28. Gulenc B, Kemah B, Yalcin S, Sayar S, Korkmaz O, Erdil M (2019) Surgical treatment of meniscal RAMP lesion. J Knee Surg. https://doi.org/10.1055/s0039-1677887

29. Hagino T, Ochiai S, Senga S, Yamashita T, Wako M, Ando T et al (2015) Meniscal tears associated with anterior cruciate ligament injury. Arch Orthop Trauma Surg 135:1701-1706

30. Hamberg P, Gillquist J, Lysholm J (1983) Suture of new and old peripheral meniscus tears. J Bone Joint Surg Am 65:193-197

31. Hash TW 2nd (2013) Magnetic resonance imaging of the knee. Sports Health 5:78-107

32. Hatayama K, Terauchi M, Saito K, Aoki J, Nonaka S, Higuchi H (2018) Magnetic resonance imaging diagnosis of medial meniscal ramp lesions in patients with anterior cruciate ligament injuries. Arthroscopy 34:1631-1637

33. Heckmann TP, Barber-Westin SD, Noyes FR (2006) Meniscal repair and transplantation: indications, techniques, rehabilitation, and clinical outcome. J Orthop Sports Phys Ther 36:795-814

34. Jan N, Sonnery-Cottet B, Fayard JM, Kajetanek C, Thaunat M (2016) Complications in posteromedial arthroscopic suture of the medial meniscus. Orthop Traumatol Surg Res 102:S287-S293

35. Keene GCR, Bickerstaff D, Rae PJ, Paterson RS (1993) The natural history of meniscal tears in anterior cruciate ligament insufficiency. Am J Sports Med 21:672-679

36. Keyhani S, Ahn JH, Verdonk R, Soleymanha M, Abbasian M (2017) Arthroscopic all-inside ramp lesion repair using the posterolateral transseptal portal view. Knee Surg Sports Traumatol Arthrosc 25:454-458

37. Kijowski R, Rosas HG, Lee KL, Cheung A, del Rio AM, Graf BK (2014) MRI characteristics of healed and unhealed peripheral vertical meniscal tears. AJR Am J Roentgenol 202:585-592

38. Kim SH, Lee SH, Kim KI, Yang JW (2018) Diagnostic accuracy of sequential arthroscopic approach for ramp lesions of the posterior horn of the medial meniscus in anterior cruciate ligament-deficient knee. Arthroscopy 34:1582-1589

39. Kim Y, Ahn JM, Kang Y, Lee E, Lee JW, Kang HS (2018) Uncovered medial meniscus sign on knee MRI: evidence of lost brake stop mechanism of the posterior horn medial meniscus. AJR Am J Roentgenol 211:1313-1318

40. Kumar NS, Spencer T, Cote MP, Arciero RA, Edgar C (2018) Is edema at the posterior medial Tibial plateau indicative of a ramp lesion? An examination of 307 patients with anterior cruciate ligament reconstruction and medial meniscal tears. Orthop J Sports Med:6

41. Lerat JL, Imbert P, Moyen B, Besse JL, Brunet-Guedj E, Bochu M (1995) Results of sutures of the internal meniscus associated with reconstruction of the anterior cruciate ligament in chronic knee joint instability. Apropos of 42 cases, 30 of them controlled by arthrography. Rev Chir Orthop Reparatrice Appar Mot 81:514-526

42. Levy IM, Torzilli PA, Warren RF (1982) The effect of medial meniscectomy on anterior-posterior motion of the knee. J Bone Joint Surg Am 64:883-888

43. Li WP, Chen Z, Song B, Yang R, Tan W (2015) The FasT-fix repair technique for ramp lesion of the medial meniscus. Knee Surg Relat Res 27:56-60

44. Liu X, Feng H, Zhang H, Hong L, Wang XS, Zhang J (2011) Arthroscopic prevalence of ramp lesion in 868 patients with anterior cruciate ligament injury. Am J Sports Med 39:832-837

45. Liu X, Zhang H, Feng H, Hong L, Wang X-s, G-y S (2017) Is it necessary to repair stable ramp lesions of the medial meniscus during anterior cruciate ligament reconstruction? A prospective randomized controlled trial. Am J Sports Med 45:1004-1011

46. Lyman S, Hidaka C, Valdez AS, Hetsroni I, Pan TJ, Do H et al (2013) Risk factors for Meniscectomy after meniscal repair. Am J Sports Med 41:2772-2778

47. Magee T, Williams D (2006) 3.0-T MRI of meniscal tears. AJR Am J Roentgenol 187:371-375

48. Malatray M, Raux S, Peltier A, Pfirrmann C, Seil R, Chotel F (2018) Ramp lesions in $\mathrm{ACL}$ deficient knees in children and adolescent population: a high prevalence confirmed in intercondylar and posteromedial exploration. Knee Surg Sports Traumatol Arthrosc 26:1074-1079

49. Mansori AEL, Lording T, Schneider A, Dumas R, Servien E, Lustig S (2018) Incidence and patterns of meniscal tears accompanying the anterior cruciate ligament injury: possible local and generalized risk factors. Int Orthop 42:2113-2121

50. Moher D, Liberati A, Tetzlaff J, Altman DG (2009) Preferred reporting items for systematic reviews and meta-analyses: the PRISMA statement. BMJ 339: b2535

51. Naendrup JH, Pfeiffer TR, Chan C, Nagai K, Novaretti JV, Sheean AJ et al (2019) Effect of meniscal ramp lesion repair on knee kinematics, bony contact forces, and in situ forces in the anterior cruciate ligament. Am J Sports Med 47:3195-3202

52. Nam TS, Kim MK, Ahn JH (2014) Efficacy of magnetic resonance imaging evaluation for meniscal tear in acute anterior cruciate ligament injuries. Arthroscopy 30:475-482

53. Oei EHG, Nikken JJ, Verstijnen ACM, Ginai AZ, Hunink MGM (2003) MR imaging of the Menisci and cruciate ligaments: a systematic review. Radiology 226:837-848

54. Peltier A, Lording TD, Lustig S, Servien E, Maubisson L, Neyret P (2015) Posteromedial meniscal tears may be missed during anterior cruciate ligament reconstruction. Arthroscopy 31:691-698

55. Pfeiffer TP, Murphy Cl, Arner JW, Musahl V (2017) Identification and treatment of RAMP lesions in anterior cruciate ligament-injured knees. Ann Jt:2

56. Phelan N, Rowland P, Galvin R, O'Byrne JM (2016) A systematic review and meta-analysis of the diagnostic accuracy of MRI for suspected ACL and meniscal tears of the knee. Knee Surg Sports Traumatol Arthrosc 24:15251539

57. Pujol N, Beaufils P (2009) Healing results of meniscal tears left in situ during anterior cruciate ligament reconstruction: a review of clinical studies. Knee Surg Sports Traumatol Arthrosc 17:396-401

58. Pujol N, Beaufils $P$ (2016) During ACL reconstruction, small asymptomatic meniscal lesions can be left untreated: a systematic review. J ISAKOS 1:135-140

59. Ramnath RR, Magee T, Wasudev N, Murrah R (2006) Accuracy of 3-T MRI using fast spin-echo technique to detect meniscal tears of the knee. AJR Am J Roentgenol 187:221-225

60. Reider B (2017) Ramped up. Am J Sports Med 45:1001-1003

61. Reitsma JB, Rutjes AWS, Whiting P, Vlassov W, Leeflang MM, Deeks JJ. Chapter 9: Assessing methodological quality. In: Deeks JJ, Bossuyt PM, Gatsonis C, eds. Cochrane Handbook for Systematic Reviews of Diagnostic Test Accuracy Version 1.0.0.: The Cochrane Collaboration; 2009

62. Rosenberg LS, Sherman MF (1992) Meniscal injury in the anterior cruciatedeficient knee. Sports Med 13:423-432

63. Rosso F, Bonasia D, Cottino U, Dettoni F, Bruzzone M, Rossi R (2018) Ramp lesion: from epidemiology to surgical treatment. Minerva Ortop Traumatol 69:55-62

64. Seil R, Hoffmann A, Scheffler S, Theisen D, Mouton C, Pape D (2017) Ramp lesions : tips and tricks in diagnostics and therapy. Orthopade 46 846-854

65. Seil R, Mouton C, Coquay J, Hoffmann A, Nuhrenborger C, Pape D et al (2018) Ramp lesions associated with ACL injuries are more likely to be present in contact injuries and complete ACL tears. Knee Surg Sports Traumatol Arthrosc 26:1080-1085

66. Shelbourne KD, Benner RW, Gray T (2017) Results of anterior cruciate ligament reconstruction with patellar tendon autografts: objective factors associated with the development of osteoarthritis at 20 to 33 years after surgery. Am J Sports Med 45:2730-2738

67. Shelbourne KD, Benner RW, Nixon RA, Gray T (2015) Evaluation of peripheral vertical nondegenerative medial meniscus tears treated with trephination alone at the time of anterior cruciate ligament reconstruction. Arthroscopy 31:2411-2416

68. Shelbourne KD, Rask BP (2001) The sequelae of salvaged nondegenerative peripheral vertical medial meniscus tears with anterior cruciate ligament reconstruction. Arthroscopy 17:270-274

69. Slim K, Nini E, Forestier D, Kwiatkowski F, Panis Y, Chipponi J (2003) Methodological index for non-randomized studies (MINORS): development and validation of a new instrument. ANZ J Surg 73:712-716

70. Smith JP 3rd, Barrett GR (2001) Medial and lateral meniscal tear patterns in anterior cruciate ligament-deficient knees. A prospective analysis of 575 tears. Am J Sports Med 29:415-419

71. G-y S, Liu X, Zhang H, Wang Q-q, Zhang J, Li Y et al (2016) Increased medial meniscal slope is associated with greater risk of ramp lesion in noncontact anterior cruciate ligament injury. Am J Sports Med 44:2039-2046

72. Sonnery-Cottet B, Conteduca J, Thaunat M, Gunepin FX, Seil R (2014) Hidden lesions of the posterior horn of the medial meniscus: a systematic arthroscopic exploration of the concealed portion of the knee. Am J Sports Med 42:921-926

73. Sonnery-Cottet B, Praz C, Rosenstiel N, Blakeney WG, Ouanezar H, Kandhari $\checkmark$ et al (2018) Epidemiological evaluation of meniscal ramp lesions in 3214 anterior cruciate ligament-injured knees from the SANTI study group database: a risk factor analysis and study of secondary Meniscectomy rates following 769 ramp repairs. Am J Sports Med 46:3189-3197 
74. Sonnery-Cottet B, Saithna A, Blakeney WG, Ouanezar H, Borade A, Daggett $M$ et al (2018) Anterolateral ligament reconstruction protects the repaired medial meniscus a comparative study of 383 anterior cruciate ligament reconstructions from the SANTI study group with a minimum follow-up of 2 years. AJR Am J Roentgenol 46:1819-1826

75. Sonnery-Cottet B, Serra Cruz R, Vieira TD, Goes RA, Saithna A (2020) Ramp lesions: an unrecognized posteromedial instability? Clin Sports Med 39:69-81

76. Stephen JM, Halewood C, Kittl C, Bollen SR, Williams A, Amis AA (2016) Posteromedial Meniscocapsular lesions increase Tibiofemoral joint laxity with anterior cruciate ligament deficiency, and their repair reduces laxity. Am J Sports Med 44:400-408

77. Menisci SM (1988) Manual of arthroscopic surgery. Springer, New York, pp $171-178$

78. Sullivan D, Levy IM, Sheskier S, Torzilli PA, Warren RF (1984) Medial restraints to anterior-posterior motion of the knee. J Bone Joint Surg Am 66:930-936

79. Thaunat M, Fayard JM, Guimaraes TM, Jan N, Murphy CG, Sonnery-Cottet B (2016) Classification and surgical repair of ramp lesions of the medial meniscus. Arthrosc Tech 5:e871-e875

80. Thaunat M, Jan N, Fayard JM, Kajetanek C, Murphy CG, Pupim B et al (2016) Repair of meniscal ramp lesions through a posteromedial portal during anterior cruciate ligament reconstruction: outcome study with a minimum 2-year follow-up. Arthroscopy 32:2269-2277

81. Van Dyck P, Vanhoenacker FM, Lambrecht V, Wouters K, Gielen JL, Dossche L et al (2013) Prospective comparison of 1.5 and 3.0-T MRI for evaluating the knee menisci and ACL. J Bone Joint Surg Am 95:916-924

82. Warren RF, Levy IM (1983) Meniscal lesions associated with anterior cruciate ligament injury. Clin Orthop Relat res 32-37

83. Wyatt RWB, Inacio MCS, Bellevue KD, Schepps AL, Maletis GB (2017) Isolated $A C L$ versus multiple knee ligament injury: associations with patient characteristics, cartilage status, and meniscal tears identified during $\mathrm{ACL}$ reconstruction. Phys Sportsmed 45:323-328

84. Yagishita K, Muneta T, Ogiuchi T, Sekiya I, Shinomiya K (2004) Healing potential of meniscal tears without repair in knees with anterior cruciate ligament reconstruction. Am J Sports Med 32:1953-1961

85. Yang J, Guan K, Wang JZ (2017) Clinical study on the arthroscopic refreshing treatment of anterior cruciate ligament injury combined with stable medial meniscus ramp injury. J Musculoskelet Neuronal Interact 17: $108-113$

86. Yeo Y, Ahn JM, Kim H, Kang Y, Lee E, Lee JW et al (2018) MR evaluation of the meniscal ramp lesion in patients with anterior cruciate ligament tear. Skelet Radiol 47:1683-1689

\section{Publisher's Note}

Springer Nature remains neutral with regard to jurisdictional claims in published maps and institutional affiliations.

\section{Submit your manuscript to a SpringerOpen ${ }^{\circ}$ journal and benefit from:}

- Convenient online submission

- Rigorous peer review

- Open access: articles freely available online

- High visibility within the field

- Retaining the copyright to your article

Submit your next manuscript at $\boldsymbol{\nabla}$ springeropen.com 